\title{
Review of physics and applications of relativistic plasmas driven by ultra-intense lasers*
}

\author{
Donald Umstadter ${ }^{\dagger, a)}$ \\ Center for Ultrafast Optical Science, University of Michigan, Ann Arbor, Michigan 48109-2099
}

(Received 26 October 2000; accepted 20 February 2001)

\begin{abstract}
As tabletop lasers continue to reach record levels of peak power, the interaction of light with matter has crossed a new threshold, in which plasma electrons at the laser focus oscillate at relativistic velocities. The highest forces ever exerted by light have been used to accelerate beams of electrons and protons to energies of a million volts in distances of only microns. Not only is this acceleration gradient up to a thousand times greater than in radio-frequency-based sources, but the transverse emittance of the particle beams is comparable or lower. Additionally, laser-based accelerators have been demonstrated to work at a repetition rate of $10 \mathrm{~Hz}$, an improvement of a factor of 1000 over their best performance of just a couple of years ago. Anticipated improvements in energy spread may allow these novel compact laser-based radiation sources to be useful someday for cancer radiotherapy and as injectors into conventional accelerators, which are critical tools for x-ray and nuclear physics research. They might also be used as a spark to ignite controlled thermonuclear fusion. The ultrashort pulse duration of these particle bursts and the $\mathrm{x}$ rays they can produce, hold great promise as well to resolve chemical, biological or physical reactions on ultrafast (femtosecond) time scales and on the spatial scale of atoms. Even laser-accelerated protons are soon expected to become relativistic. The dense electron-positron plasmas and vast array of nuclear reactions predicted to occur in this case might even help bring astrophysical phenomena down to Earth, into university laboratories. This paper reviews the many recent advances in this emerging discipline, called high-field science. (C) 2001 American Institute of Physics.
\end{abstract}

[DOI: $10.1063 / 1.1364515$ ]

\section{INTRODUCTION}

For almost 40 years, scientists have studied nonlinear optics of electrons that are bound to atoms and molecules. Nonlinear effects arise in this case due to the anharmonic oscillatory motion of electrons in the combined fields of atom and laser. The study of nonlinear optics was enabled by the invention of $Q$-switched lasers, which resulted in an orders-of-magnitude increase in the peak power of lasers. The rate of advancement in our understanding of nonlinear optics has been quite rapid. For instance, harmonic generation (e.g., frequency doubling) went from being barely detectable to almost unity efficiency in just a few years. Now the field is quite mature, having spawned numerous new research tools, subfields, and commercial products.

In the last decade, table-top lasers have undergone a similar orders-of-magnitude jump in peak power (see Fig. 1). Shorter (femtosecond) pulses, increased power, decreased size and increased repetition rate were all enabled by the use of chirped-pulse amplification ${ }^{1}$ in solid-state lasers. To avoid damage to amplifiers, laser light is stretched in time before being amplified and then recompressed. These systems now have multiterawatt peak powers and can be focused to intensities of $I \geqslant 10^{19} \mathrm{~W} / \mathrm{cm}^{2}$, which is high enough to cause nonlinearity in even unbound (free) electrons. The nonlinearity

*Paper LR1, Bull. Am. Phys. Soc. 45, 212 (2000).

${ }^{\dagger}$ Invited speaker.

${ }^{a)}$ Electronic mail: dpu@umich.edu arises in this case because the electrons oscillate at relativistic velocities in laser fields that exceed $10^{11} \mathrm{~V} / \mathrm{cm}$, resulting in relativistic mass changes exceeding the electron rest mass and the light's magnetic field becomes important. The work done by the electromagnetic field $(E)$ on an electron $(e E \lambda)$ over the distance of a laser wavelength $(\lambda)$ then approaches the electron rest mass energy $\left(m_{0} c^{2}\right)$, where $e$ is the elementary charge of an electron, $m_{0}$ is the electron rest mass, and $c$ is the speed of light. Thus, a new field of nonlinear optics, that of relativistic electrons, has been launched. As can be seen from the following review, effects analogous to those studied with conventional nonlinear optics-self-focusing, self-phase-modulation, harmonic generation, and so on-are all found, but based on this entirely different physical mechanism. Rapid advancement is underway and new research tools, subfields, and commercial products are on the horizon, e.g., compact and ultrashort pulse duration laserbased electron accelerators and x-ray sources.

We look forward to the next physical regime that will be encountered at even higher intensities $\left(I \simeq 10^{24} \mathrm{~W} / \mathrm{cm}^{2}\right)$ where even protons will quiver relativistically, i.e., the work done on a proton over the distance of a laser wavelength approaches its rest mass energy. This might be called the nuclear regime of laser-plasma interactions, because of the fusion and fission reactions and the generation of pions, muons, and neutrinos that should occur as nuclei collide in such energetic plasmas.

This review will discuss the latest developments in this 


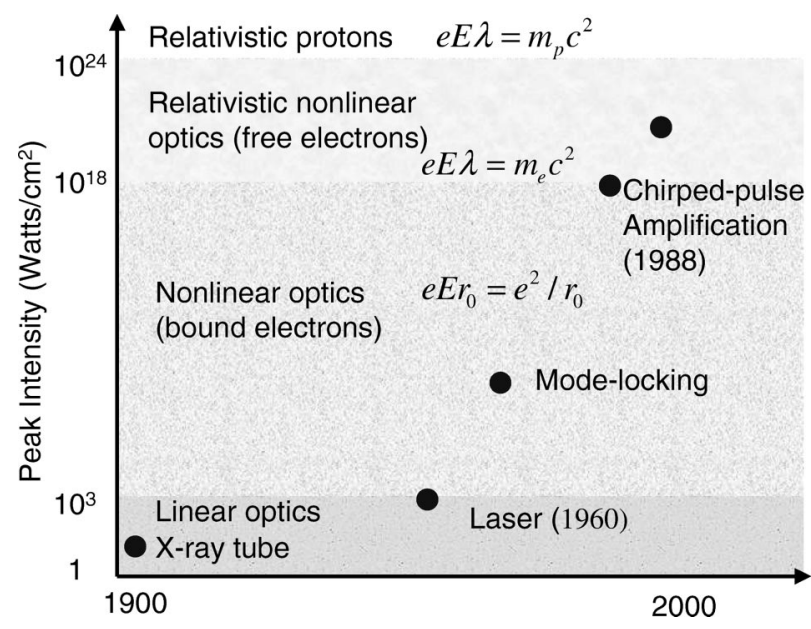

FIG. 1. History of light sources over the last century. Each advance in laser intensity (corresponding in this figure to visible or infrared light) enables a new regime of optics.

new field of physics, known as high-field science. It is not intended to be comprehensive, but rather to be restricted to a discussion of some of the highlights, mainly over the last 5 years, in the relativistic regime of laser-plasma interactions. Several reviews have already been published on highintensity laser development and applications, ${ }^{2-4}$ relativistic nonlinear optics, ${ }^{4,5}$ laser accelerators, ${ }^{6}$ and intense laserplasma interactions. ${ }^{7-12}$

The paper is organized as follows. A brief basic theoretical overview of relativistic laser-plasmas interactions, with references only to early work, is presented in Sec. II. Recent results and references to more recent theoretical and numerical work are discussed in Sec. III A; experimental results are presented in Sec. III B. Prospects and applications are reviewed in Sec. IV.

\section{BRIEF OVERVIEW}

\section{A. Underdense plasma}

\section{Electron quiver motion}

When an intense laser pulse is focused on a supersonic flow of gas with a sharp gas-vacuum interface and the field strength ramps up, the interaction physics passes through the different physical regimes discussed in Sec. I. At the beginning of the pulse, when the field strength is low, atomic electrons oscillate in the electromagnetic field at the laser frequency $\left(\omega_{0}=2 \pi c / \lambda=c k\right)$. Perturbation theory breaks down when the work done on an electron $\left(e E r_{0}\right)$ over the distance of the Bohr radius $\left(r_{0}\right)$ approaches the Coulomb potential energy $\left(e^{2} / r_{0}\right)$. At field strengths that reach the multiphoton or tunnelling ionization intensity, the electrons become stripped from the atoms, i.e., the gas becomes ionized. At even higher intensities, the liberated electrons quiver at velocities close to the speed of light $(c)$, the relativistic electron mass increases, and the $v \times B$ force in the Lorentz equation of motion [Eq. (1)] becomes important

$$
F=\frac{d\left(\gamma p_{0}\right)}{d t}=e E+\left(\frac{e}{c}\right)(v \times B) .
$$

In the relativistic regime, the quiver momentum of the electrons, $p_{0}$, exceeds $m_{0} c$. The ratio of these two momenta is specified by the parameter $a_{0}$, the normalized vector potential, defined as $a_{0}=p_{0} / m_{0} c=e E / m_{0} \omega_{0} c$, where $E$ is the electric field amplitude of the laser light. In practical units, $a_{0}=0.85 \times 10^{-9} \sqrt{I} \lambda$, where $I$ is the intensity of the laser light in $\mathrm{W} / \mathrm{cm}^{2}$ and $\lambda$ is the wavelength of the laser light in microns. The electron mass $m_{e}$ begins to change significantly compared to the electron rest mass when $a_{0} \simeq 1$, which is satisfied for $1 \mu \mathrm{m}$ light at a laser intensity of $\sim 10^{18} \mathrm{~W} / \mathrm{cm}^{2}$. The relativistic regime was actually first approached as early as the late 1970s with large $\mathrm{CO}_{2}$ lasers operating at $10 \mu \mathrm{m}$ wavelength and reaching intensities of $10^{15} \mathrm{~W} / \mathrm{cm}^{2}$, corresponding to $a_{0} \simeq 0.3$. $^{13}$ Terawatt power $\mathrm{CO}_{2}$ lasers have now been developed, which employ solid-state switches. ${ }^{14,15}$

As predicted by Eq. (1), the motion of an electron that becomes relativistic in an intense field is described by a figure eight lying along the plane defined by the polarization vector and $\hat{k}$, rather than a straight line along the polarization vector (as in the case of low-intensity light). This originates from the fact that $v \times B \propto E \times B \propto E^{2} \hat{k}$. According to customary thinking, electrons that move in this way should radiate photons at harmonics of the frequency of the incident laser light, with each harmonic having its own unique angular distribution. This is referred to as nonlinear Thomson scattering or relativistic Thomson scattering, predicted over 50 years ago. $^{16}$

Quantum electrodynamic effects, such as pair production from the vacuum, will become important when the work done by the laser electric field over a distance of a Compton wavelength $\left(\lambda_{C} \equiv \hbar / m c\right)$ equals the rest mass of an electron. This requires that $E_{0}$ equal a value that will be difficult to achieve even in the foreseeable future $\left(3 \times 10^{16} \mathrm{~V} / \mathrm{cm}\right.$ or $I$ $\left.=10^{30} \mathrm{~W} / \mathrm{cm}^{2}\right)$. Although the field strength required for the catastrophic production of electron-positron pairs from the vacuum is six orders of magnitude higher than is currently available with even the most intense lasers, the observation of a statistically significant number of pairs was shown to occur at fields as low as $E_{0} \sim 10^{14} \mathrm{~V} / \mathrm{cm}^{17}$

\section{Media effects}

The dielectric properties of a plasma medium are also affected as the relativistic change in the electron mass alters the plasma frequency, $\omega_{p}=\omega_{p 0} / \gamma^{1 / 2}=\left(4 \pi n_{e} e^{2} / \gamma m_{0}\right)^{1 / 2}$, where $\omega_{p 0}$ is the plasma frequency in a quiescent plasma, $n_{e}$ is the plasma electron density and $\gamma=\sqrt{1+a_{0}^{2}}$ is the relativistic Lorentz factor. This change in plasma frequency in turn modifies the index of refraction of the light wave, which is given by $\eta=\left[1-\left(\omega_{p} / \omega_{0}\right)^{2}\right]^{1 / 2}$. Thus, an on-axis maximum of $\eta(r)$ can be created through modification of the radial profile of $\gamma$ and/or $n_{e}$. For optical guiding of laser pulses in underdense plasmas $\left(\omega_{0}>\omega_{p}\right)$, the radial profile of the index of refraction, $\eta(r)$, must have a maximum on axis, causing the wavefront to curve inward and the laser beam to converge. A situation in which $\gamma(0)>\gamma(r)$ can be created by a laser beam with an intensity profile peaked on axis, as shown in Fig. 2. When this focusing effect just balances the defocusing caused by diffraction, the laser pulse can propagate 


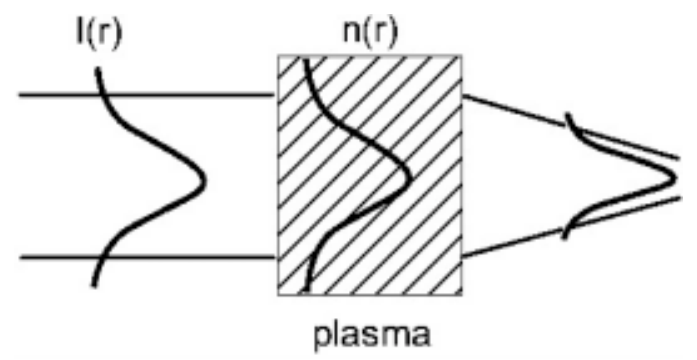

FIG. 2. Relativistic self-focusing occurs when the index of refraction has a radial maximum on-axis due to a relativistic mass change caused by an on-axis peak in the laser intensity.

over a longer distance than it could in vacuum, while maintaining a small cross section. This mechanism is referred to as relativistic self-guiding and should occur provided the laser power exceeds a critical power given by $P_{c}$ $=17\left(\omega_{0} / \omega_{p}\right)^{2} \mathrm{GW} .{ }^{18,19}$ For plasmas created by photoionization of a gas with a laser pulse that is spatially shaped like a Gaussian in the transverse dimension, the density will be higher on the axis than off the axis. The refractive index will thus be lower on axis, which will tend to defocus the light and increase the self-guiding threshold. For this reason, low$Z$ gases with few ionization stages, such as $\mathrm{H}_{2}$, are commonly used as targets.

\section{Ponderomotive pressure}

Any spatial variation of the laser intensity will act to push electrons to regions of lower intensity through the socalled ponderomotive force, which for $a_{0} \ll 1$ is proportional to the gradient of the light pressure, $\nabla P \propto \nabla\left(n_{e} I \lambda^{2}\right)$, where the light pressure is the time-averaged quiver energy density, $P=n_{e}(\gamma-1) m_{0} c^{2}$. In the highly relativistic regime, $a_{0} \gg 1$ (e.g., $10^{20} \mathrm{~W} / \mathrm{cm}^{2}$ for $1 \mu \mathrm{m}$ light), the light pressure at solid density $\left(10^{24} \mathrm{~cm}^{-3}\right)$ can approach the thermal pressure $\left(n k T_{e}\right)$ of the Sun's core (250 billion atmospheres), where $n$ is $10^{25} \mathrm{~cm}^{-3}$.

A Gaussian-shaped laser's ponderomotive force will tend to expel electrons radially from the region of the axis, so-called "electron cavitation." If the ponderomotive force is high enough for long enough, the charge displacement due to expelled electrons will eventually cause the ions to move as well, forming an on-axis density channel. Because $n_{e}(0)$ $<n_{e}(r)$, and thus $\eta(0)>\eta(r)$, this enhances the previously discussed relativistic self-guiding and can itself guide a laser pulse. $^{20-22}$

Since the laser phase velocity $v_{\phi}$ depends on the index of refraction, $v_{\phi}=c / \eta$, it will then depend on the laser intensity. Local variation in the phase velocity will modify the shape of the laser pulse, and, consequently, the spatial and temporal profile of the laser intensity, which then further changes locally the index of refraction and so on. This modulation of light intensity, coupled to the ponderomotive force and self-focusing, can lead to self-modulation, Raman scattering, etc. ${ }^{23-25}$

\section{Electron acceleration}

For time periods that are short compared to an ion period, electrons are displaced from regions of high laser intensity, but ions, due to their much greater inertia, remain stationary. The resulting charge displacement provides an electrostatic restoring force that causes the plasma electrons to oscillate at the plasma frequency $\left(\omega_{p}\right)$ after the laser pulse passes by them, creating alternating regions of net positive and negative charge. The resulting electrostatic wakefield plasma wave propagates at a phase velocity nearly equal to the speed of light and thus can continuously accelerate properly phased electrons.

Acceleration of electrons by electron plasma waves is of current interest because the acceleration gradient (200 $\mathrm{GeV} / \mathrm{m}$ ) is much larger (four orders of magnitude larger) than in conventional rf linacs $(<20 \mathrm{MeV} / \mathrm{m}) .{ }^{26,6}$ Several methods have been proposed for driving a large-amplitude high-phase-velocity plasma wave, such as the plasma wakefield accelerator, ${ }^{27}$ the plasma beatwave accelerator, ${ }^{26}$ the laser wakefield accelerator (LWFA), ${ }^{26,28}$ the resonant laser plasma accelerator, ${ }^{29}$ and the self-modulated laser wakefield accelerator (SMLWFA). ${ }^{24,25,23}$ The plasma wakefield ${ }^{30}$ and beatwave accelerators ${ }^{31-33}$ were demonstrated first because the required technologies, relativistic electron beams in the former case or long-pulse medium-power lasers in the latter case, were well developed. More recently, the LWFA and the SMLWFA have received considerable attention and shown rapid progress because of the development of table-top ultrashort-duration terawatt-peak-power lasers. In the SMLWFA, an electromagnetic wave $\left(\omega_{0}, \mathbf{k}_{\mathbf{0}}\right)$ decays into a plasma wave $\left(\omega_{p}, \mathbf{k}_{\mathbf{p}}\right)$ and another forward-propagating light wave $\left(\omega_{0}-\omega_{p}, \mathbf{k}_{\mathbf{o}}-\mathbf{k}_{\mathbf{p}}\right)$ via the stimulated Raman forwardscattering instability. In this case, the laser pulse duration is longer than an electron plasma period, $\tau \gg \tau_{p}=2 \pi / \omega_{p}$. In the LWFA, an electron plasma wave is driven resonantly by a short laser pulse $\left(\tau \sim \tau_{p}\right)$ through the laser ponderomotive force. In the resonant laser plasma accelerator, a train of Gaussian-shaped pulses with variable durations and interpulse spacings can stay in resonance with a wakefield as it grows nonlinearly. ${ }^{29}$

The injection of electrons into plasma waves can occur uncontrollably by trapping of hot background electrons, which are preheated by other processes such as Raman backscattering and sidescattering instabilities, ${ }^{34}$ or by wave breaking (longitudinal $^{35}$ or transverse ${ }^{37}$ ). Because the electrons in this case are injected into the plasma wave uniformly in phase space, large energy spreads result, as is typically observed in the SMLWFA regime. ${ }^{35}$ The injection can be controlled by use of an external electron source (such as from an RF gun); ${ }^{32,33}$ however, because the pulse durations of the injected electron bunches in the experiments in which this method was tried were longer than the acceleration buckets, the energy spread was again large. It has been shown analytically and numerically that controlled injection might also be accomplished by means of internal electrons, from the plasma itself, which are all put into the accelerating phase of the plasma wave by a separate laser pulse. ${ }^{38}$ Such a laser-driven plasma-cathode electron gun might eventually 
have (1) monoenergetic energy, (2) GeV/cm acceleration fields, (3) micron source size, (4) femtosecond pulse duration, (5) high brightness, (6) absolute synchronization between electrons and laser (for pump and probe experiments), and (7) compact size (university-lab scale).

\section{Self-generated magnetic fields}

In intense laser-plasma interactions, several mechanisms can result in the self-generated quasistatic magnetic fields of megaGauss amplitude. For instance, the electron beam discussed in Sec. II A 4 will drive an axial current, which can result in a toroidal magnetic field. ${ }^{39}$ The thermoelectric effect can also induce a toroidal field. The inverse Faraday effect, in which a circularly polarized light beam induces current loops, will generate an axial magnetic field. ${ }^{40}$

\section{B. Overdense plasma}

We discuss in this section interactions with targets that are solid instead of gaseous density. In this case, lowintensity laser light $\left(a_{0} \ll 1\right)$ cannot propagate beyond the location of the critical density, $n_{c}$, defined by the density at which $\omega_{0}=\omega_{p}$.

\section{Proton acceleration}

Plasma ions can be accelerated to high energies by the formation of an electrostatic sheath due to charge displacement. The latter results from the initial preferential acceleration of electrons; the heavier ions are left behind due to inertia. Among the many mechanisms that can accelerate the electrons are: thermal expansion, plasma waves, " $J \times B$ heating" or "vacuum heating." 41 Energetic ions have been accelerated by thermal expansion in long-pulse (low power) laser-plasma experiments for over a decade. The latter two mechanisms, however, are unique to high-intensity lasers and originate from the instantaneous ponderomotive force, which (for $a_{0} \ll 1$ ) has a frequency twice that of the pump and a magnitude proportional to $a_{0}^{2}$. When light encounters a sharp interface between vacuum and solid density, the electromagnetic field becomes evanescent in the region above the critical density. Thus electrons gain relativistic energies as they can only complete half of their figure-eight orbits, on the vacuum side. They continue to move through the overdense region, without the laser electromagnetic field to pull them back. A nonequilibrium electrostatic sheath can thus form, which will accelerate the ions left behind. Ions will also be accelerated by each other's unshielded charges in what has been termed a "Coulomb explosion." 42

\section{Harmonic generation from the critical surface}

As has been observed since the early days of intense laser interactions (with $\mathrm{CO}_{2}$ lasers), the critical surface (the location of the critical density) can oscillate at $2 \omega$ due to the effect of the ponderomotive force, which has been used to explain the excitation of harmonics in solid-target experiments. ${ }^{43}$ Relativistic mass shifts can also shift the critical density to higher values, inducing transparency of solid targets to intense pulses. Intense laser pressure can push the

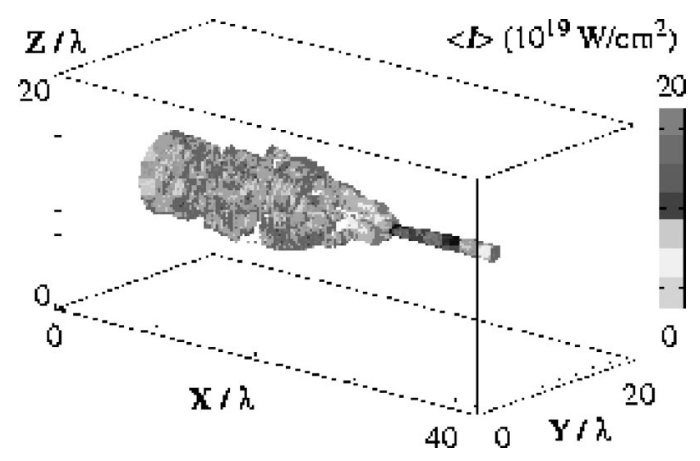

FIG. 3. 3D PIC-code simulation of relativistic self-focusing in a plasma.

critical surface towards the solid-density region ${ }^{44,45}$ and push plasma sideways in a process called "hole boring." 46

\section{RECENT RESULTS}

\section{A. Theoretical and numerical}

The one-dimensional (1D) nonlinear regime of intense laser interactions with underdense plasma is very well understood theoretically, including nonlinear plasma waves, wave breaking, quasistatic laser propagation, nonlinear growth rates for instabilities and harmonic generation. There are also numerous fluid, particle, and Vlasov codes. The 3D linear regime $\left(a_{0}^{2} \ll 1\right)$ is also well understood, including wakefield generation, relativistic self-focusing, self-channeling, selfmodulation, linear growth rates for instabilities, pulse propagation in channels, and harmonic generation.

In the 3D nonlinear regime, a unified cold-fluid-Maxwell model treatment of electron parametric instabilities driven by ultraintense laser light in plasma has recently been presented, assuming a 1D plane wave pump laser field. ${ }^{47,48} \mathrm{~A}$ new class of whole-beam instabilities, which include the effects of a radially bounded pump laser field, have also been analyzed, ${ }^{49,50}$ which describe novel effects such as the coupling of forward Raman and self-modulation instabilities. In addition to theoretical studies, a great deal of progress has been made in this regime through simulations..

2D-3D full-scale particle-in-cell (PIC) simulations are very demanding computationally, but make no approximations to the equations being solved other than those inherent in the numerical method. In this case, the equation of motion, Eq. (1), and Maxwell's equations, $\partial_{t} E=c \boldsymbol{\nabla} \times B-4 \pi j$ and $\partial_{t} B=-c \boldsymbol{\nabla} \times E$, are solved for each particle simultaneously. An example is the Virtual Laser Plasma Laboratory (VLPL), which can run on a massively parallel computer CRAY-T3E with 784 processor elements. The simulation uses up to $10^{9}$ particles and $10^{8}$ grid cells. This code was used to observe relativistic self-focusing and cavitation, ${ }^{51}$ as shown in Fig. 3, and later hole boring. ${ }^{52}$ The coalescence of two filaments into a single filament was thought to be due to the action of a self-generated magnetic field.

OSIRIS, a 3D fully explicit, object-oriented, parallel PIC code, was recently used to observe that two co-propagating laser beams in a plasma appear to form a braided pattern due to their mutual attraction. ${ }^{53}$ The modification of the index of refraction caused by one filament affects the propagation of 


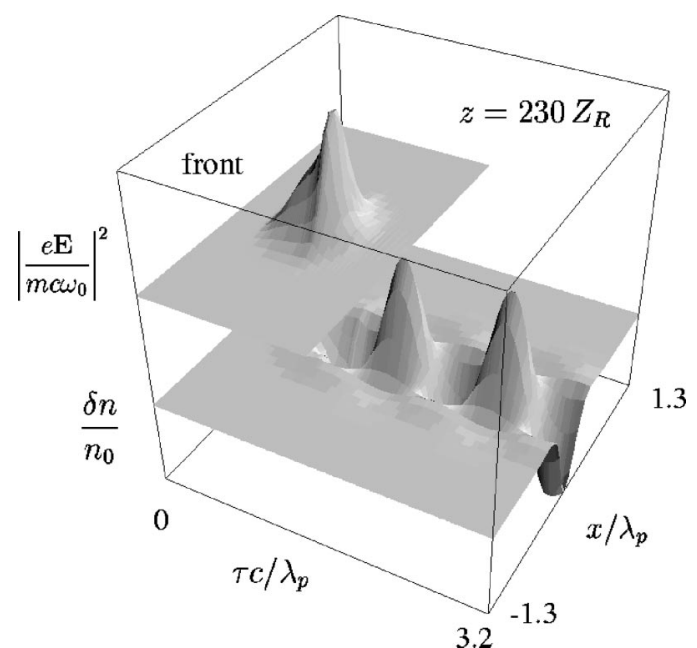

FIG. 4. The "standard" resonant wakefield simulated with SIMLAC, a numerical code in which the simulation box moves with the light pulse at its group velocity.

the other. Theory shows that in a plasma the centroids of two laser beams behave just like two point masses under mutual attraction, with the laser power playing the role of mass.

The SIMLAC code has been used to study wakefield generation and laser propagation in the limit $a^{2} \ll 1{ }^{54}$ It draws from nonlinear optics models and treats propagation in the group velocity frame. In this idealized model (which assumes perfect Gaussian beams), the pulse and wake, shown in Fig. 4, are maintained over long enough propagation distance to accelerate an electron to GeV energy. Selfmodulation has also been modelled with a 2D envelope model that does not make the paraxial approximation and thus allows for a wave with a finite group velocity, which is found to reduce the growth rate of the SMLWFA. ${ }^{49} \mathrm{~A}$ Maxwell-fluid model that does not assume a separation of the $\omega$ and $\omega_{p}$ time scales has also been studied, which for intense pulses is found to be valid in the very-underdenseplasma limit. ${ }^{55}$ A multiple time scale expression for the ponderomotive force of an intense light pulse has also been proposed. ${ }^{56}$ PIC-code simulations have been used to show that self-focusing and ponderomotive blow-out can be suppressed by the occurrence of Raman scattering and plasma heating. ${ }^{59}$ An instability that results in the generation of forward Raman radiation shifted by half the plasma frequency has been investigated analytically. ${ }^{58}$

It has been shown theoretically that non-Gaussianshaped pulses can drive wakefields more effectively than Gaussian-shaped pulses. ${ }^{60}$ Similar improvements might be obtained by the use of pulse shapes that are more easily produced experimentally using a genetic algorithm. ${ }^{61} \mathrm{~A}$ method to generate large wakefields using two slightly detuned counterpropagating laser beams has been studied numerically. ${ }^{57}$ Several PIC and test-particle simulations ${ }^{34,62-67}$ have been employed to study the characteristics of the electron beam accelerated by a plasma wave. The magnetic field of a plasma wake driven by laser pulse has also been studied. ${ }^{68}$ A $2 \mathrm{D}$ PIC code was used to study the propagation of an intense pulse through an underdense plasma, showing the formation of a "shock" on the front of the pulse, ion filaments, and double layers. ${ }^{69}$ The amplitude of the circular polarized laser was $a_{0}=50$, the mass ratio was $m_{p} / m_{e}=1840$, and $\omega_{p} / \omega_{0}=0.45$.

Several variations of the all-optical electron injection concept $^{38}$ have been studied numerically and analytically. ${ }^{69-73}$ In the original concept, ${ }^{38}$ electrons were accelerated ponderomotively by a separate laser pulse, with the electrons either coming from the plasma or created by photoionization. For greater control, it has been suggested that two injection pulses could beat together to inject electrons more locally in phase space than one pulse. ${ }^{70}$ In another study, the injection pulse could also be made to drive its own wake, which could then collide with the primary wake. ${ }^{72}$ Other ideas that have been investigated are the use of ionization in a separate gas jet, ${ }^{73}$ single cycle laser pulses, ${ }^{71}$ and density jumps. ${ }^{69}$

A 2D version of the VLPL code has been used to investigate electron acceleration by the inverse-free-electron-laser mechanism. ${ }^{74}$ Electrons propagating in a plasma channel can execute betatron oscillations from the self-generated static electric and magnetic fields. If the power of the laser greatly exceeds the threshold for relativistic self-focusing and the betatron oscillations are in resonance with the light pulse's electric field, then the electrons can gain energy directly from the laser. The results of a simulation of electron acceleration from an underdense target were used to support the argument that most of the energy acquired in a real experiment conducted under similar conditions ${ }^{75}$ (see Sec. III B 3) is due to direct laser acceleration, while laser wakefield acceleration (LWFA) drives only a minority of electrons.

Several authors have studied the acceleration of electrons directly from the laser field in vacuum. ${ }^{76,77}$

As shown in Fig. 5, a 3D PIC code was also used to simulate electrons and ions emitted from a plasma slab, ${ }^{78}$ with a maximum proton energy of $40 \mathrm{MeV}$ and electron and proton densities of $10^{21} \mathrm{~cm}^{-3}$. A PIC simulation was used to follow the particles accelerated from a plasma slab, ${ }^{79}$ showing that those protons emitted from the back side occupy a narrower longitudinal velocity phase space than those that come from the front side. A low-emittance proton beam was observed in a simulation of a laser incident on a thin foil target that was shaped like a hemisphere so that the field lines would converge on axis. ${ }^{80}$ The angular distributions of fast electrons, ions, and bremsstrahlung x-rays generated during the interaction of an ultrashort intense laser pulse with solid targets was studied analytically and with PIC code simulations. $^{81}$

PIC codes have also been used to study harmonics generated from critical-surface interactions. A normal incidence circularly polarized laser is predicted to generate harmonics with each order having a different characteristic angle. ${ }^{82-84}$

The rate of collisional absorption has been calculated when the plasma temperature is nonrelativistic and the electron quiver velocity is fully relativistic. ${ }^{85}$

The production of electron-positron pairs by the interaction of relativistic superthermal electrons, generated by ultraintense laser pulses, with high- $Z$ material has been considered. ${ }^{86}$ It is found that when the pairs are sufficiently 

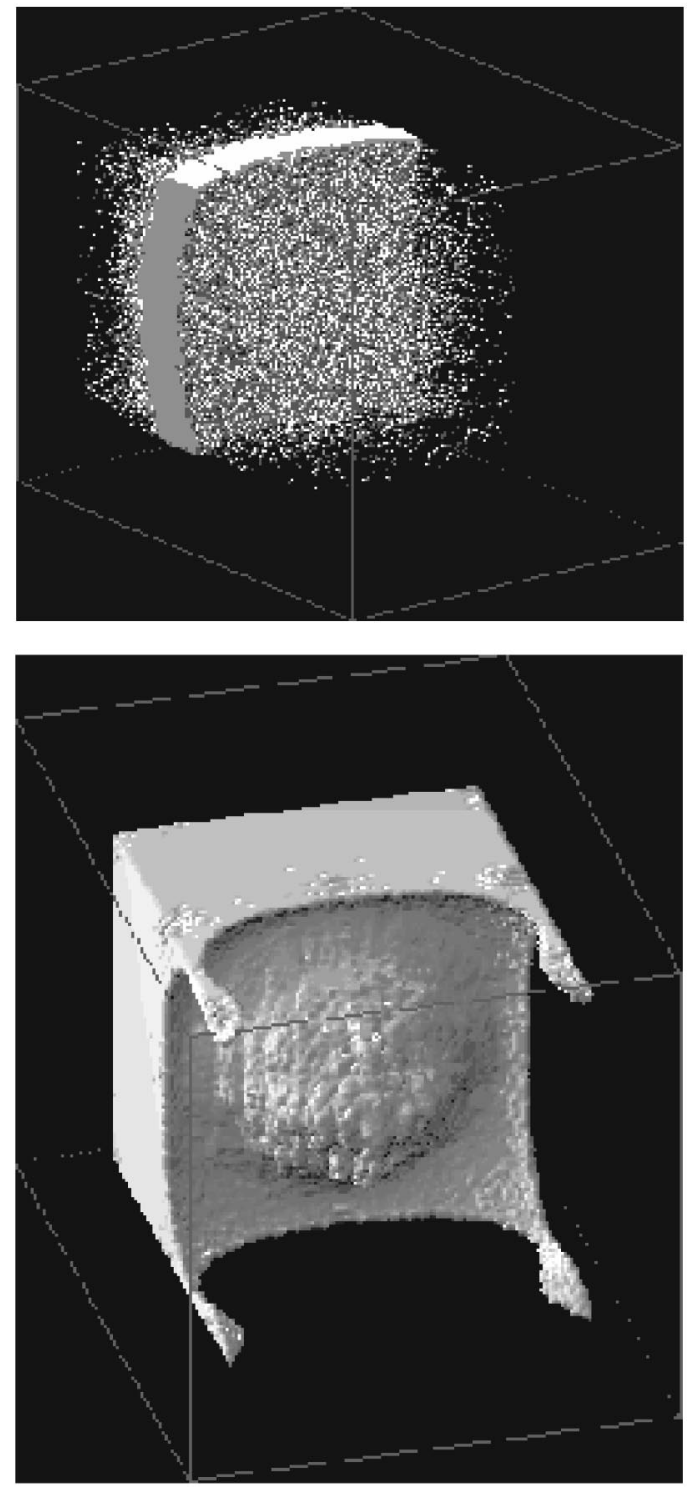

FIG. 5. A 3D PIC simulation of a laser interacting with a plasma slab shows electrons (top) streaming through the target at the Alven current and protons (bottom) up to $40 \mathrm{MeV}$.

confined, they can start to exponentiate in number, achieving a pair density approaching $10^{21} \mathrm{~cm}^{-3}$.

\section{B. Experiment}

The following experiments employed the use of solidstate laser amplifiers (either Nd:glass or Ti:sapphire) that produced infrared light (either $1.05 \mu \mathrm{m}$ or $0.8 \mu \mathrm{m}$ ) with ultrashort pulse durations (either $0.5 \mathrm{ps}$ or $100 \mathrm{fs}$ ) and intensities ranging from $10^{17}-10^{20} \mathrm{~W} / \mathrm{cm}^{2}$.

\section{Fundamental interactions}

Recently, the unique angular distribution of the second and third harmonics emitted from nonlinear relativistic Thomson scattering has been observed experimentally (see Fig. 6) ${ }^{87}$ More recently, coherent harmonic emission in the forward direction has been observed. ${ }^{88}$ With suitable phase matching, the latter may eventually lead to the generation of coherent beams of ultrashort pulses of $\mathrm{x}$ rays.
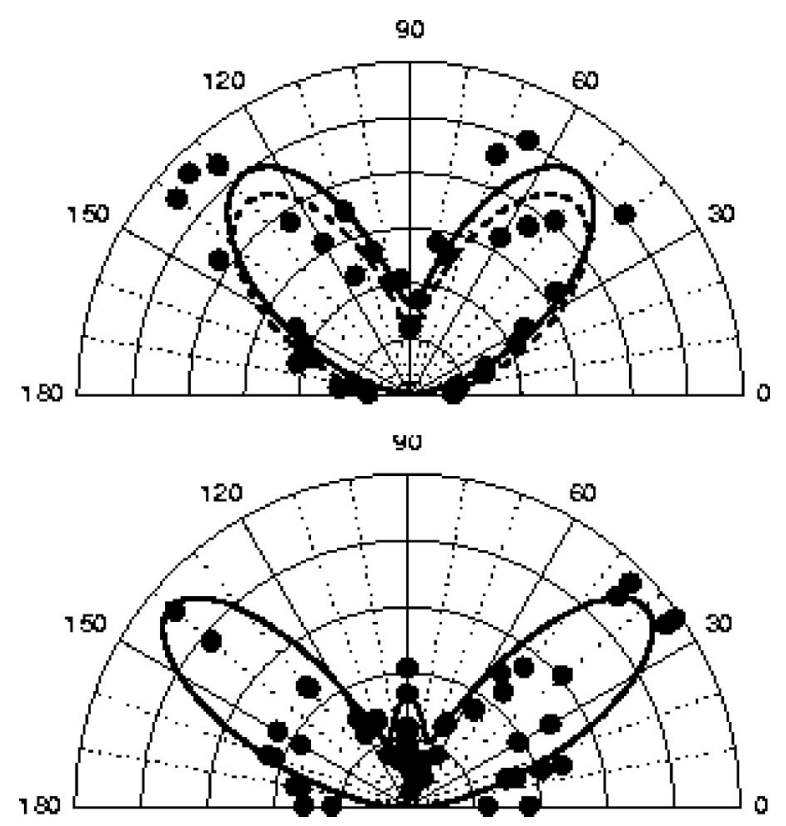

FIG. 6. Angular pattern of the second-harmonic light. Shown are polar plots of the intensity of the second-harmonic light (top) and third harmonic (bottom) as a function of azimuthal angle. Filled circles, experimental data; solid and dashed lines, theoretical results.

The conservation of canonical angular momentum has been demonstrated in experiments that studied the angular distribution of relativistic electrons emitted from barriersuppression ionization of atoms in intense laser fields. ${ }^{89}$

The generation of high harmonics created during the interaction of a $2.5 \mathrm{ps}, 1053 \mathrm{~nm}$ laser pulse with a solid target has been recorded for intensities up to $10^{19} \mathrm{~W} / \mathrm{cm}^{2}$. Harmonic orders up to the 68th at $15.5 \mathrm{~nm}$ in first-order diffraction have been observed with indications of up to the 75th at $14.0 \mathrm{~nm}$ in second-order diffraction..$^{90}$ The power of the 38th high harmonic at $27.7 \mathrm{~nm}$ is estimated to be $24 \mathrm{MW}$.

An experimentally measured increase in laser absorption was observed as the laser intensity was increased, ${ }^{91}$ which was attributed to vacuum heating, consistent with theoretical predictions. $^{41}$

The fact that the electric field in a frame moving with a relativistic electron beam is boosted by $\gamma$, where $\gamma$ is the relativistic factor associated with electron beam, has allowed the observation of pair production from the vacuum with current laser technology. ${ }^{92}$ Using the $30 \mathrm{GeV}$ electron beam of the Stanford Linear Accelerator, the field was increased by a factor of $5 \times 10^{4}$, and so in this case the threshold for observation of pair production was exceeded with a laser operating at an intensity of only $10^{19} \mathrm{~W} / \mathrm{cm}^{2}$.

\section{Guiding}

As discussed in Sec. II, self-guiding is possible when laser power exceeds the threshold for relativistic selfguiding, $P_{c}$. Various authors have reported the observation of relativistic self-guiding. ${ }^{93-98,118}$ Shown in Fig. 7 is a recent measurement, showing side-on (right) and end-on (left) imaging of Thomson scattered light for different laser powers. ${ }^{98}$ The gas jet region is $1000 \mu \mathrm{m}$ long. At low pow- 


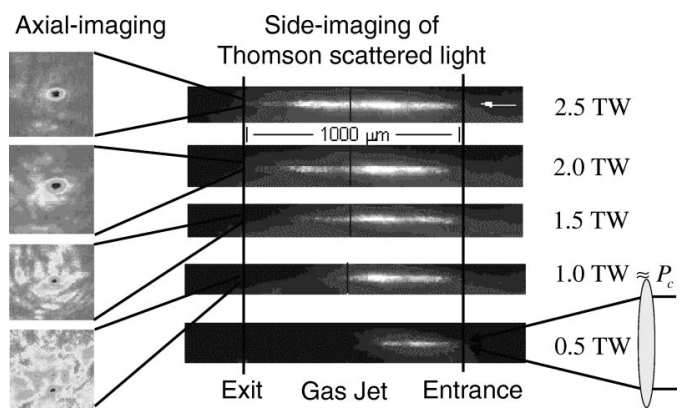

FIG. 7. Relativistic self-guiding, measured by side-on (right) and end-on (left) imaging of Thomson scattered light for different laser powers. In the former case, the laser is incident from right to left. The gas jet region is 1000 $\mu \mathrm{m}$ long. At low powers, below the relativistic self-focusing threshold, the laser propagates only a Rayleigh range, while above $P_{c}$, it extends further.

ers, below the relativistic self-focusing threshold, the laser propagates only a Rayleigh range, while at above $P_{c}$, it extends further. As can be seen from the image on the left, at the highest power, the light leaves the plasma with the same spot size as it had when it entered. Such a preformed channel has been measured interferometrically ${ }^{100}$ and a second intense laser pulse has been guided in it. ${ }^{99,100}$ Relativistic filamentation (a partial beam analog to the whole beam effect, or multiple filaments) has also been observed. ${ }^{101,102}$ Along with beam breakup, a time-dependent spectral modulation of the laser pulse transmitted through the plasma was observed using a frequency-resolved optical gating (FROG) diagnostic, indicative of motion of electrons in and out of the beam. ${ }^{101}$

The propagation of intense pulses in preformed plasmas has also been studied. For instance, an axicon lens has been used to produce a density channel, extending many Rayleigh lengths, created by means of thermal expansion driven by long-pulse channel-forming laser. ${ }^{103}$ Such channels have been used to guide pulses of intensities reaching $10^{17} \mathrm{~W} / \mathrm{cm}^{2}$ in plasmas reaching densities of $10^{16} \mathrm{~cm}^{-3}$. In order to take advantage of the uniformity of low- $Z$ gases, such as helium and hydrogen, a spark discharge was used to seed breakdown. ${ }^{104}$ Alternatively, channels can be formed by using two transversely injected laser pulses, as in the ignitorheater scheme. ${ }^{105}$ Capillary discharges have been used to guide pulses, achieving $70 \%$ transmission of pulses with intensities reaching $10^{17} \mathrm{~W} / \mathrm{cm}^{2}$ over distances of up to 20 Rayleigh ranges. ${ }^{106}$

The observation of laser self-focused channel formation into overdense plasmas (hole boring) was reported in experiments making use of a soft $\mathrm{x}$-ray laser probe system with a grid image refractometry technique. Cross sections of a 30 $\mu \mathrm{m}$ diameter channel were obtained that the authors attribute to hole boring in overdense plasmas. ${ }^{107}$ Hole boring has also been investigated by means of transmission measurements. ${ }^{108}$

\section{Electron acceleration}

Several labs have observed the acceleration of $\mathrm{MeV}$ electrons by the SMLWFA, ${ }^{35,36,98,109-112}$ sometimes accompanied by self-guiding, ${ }^{98,109}$ but with large-electron energy

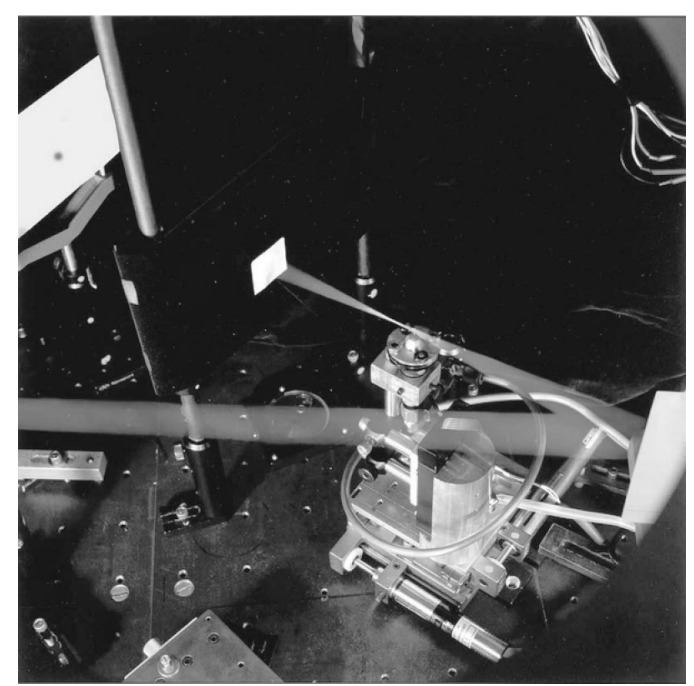

FIG. 8. Photograph of the acceleration of an electron beam by a laser interacting with a gas jet inside a vacuum chamber. The laser (illustrated for the purpose of orientation) crosses the picture from left to right and is focused by a parabolic mirror (right side of the picture). The supersonic nozzle (shown in the middle of the picture) is position with micron accuracy with a 3 -axis micropositioner. The $e$-beam makes a small spot on a white florescent (LANEX) screen, shown in the upper left-hand corner of the picture.

spreads (most of the electrons have energies less than 5 $\mathrm{MeV}$, with the number decaying exponentially with a temperature of $\sim 1 \mathrm{MeV}$ to just a few electrons at energies up to $100 \mathrm{MeV})$. The origin of the accelerated electrons is a subject of some debate. It has been attributed to catastrophic wave breaking of a relativistic Raman forward scattered plasma wave, ${ }^{35}$ or to wave breaking of slower velocity Raman backscattered waves in both experiment ${ }^{36,109}$ and theory. ${ }^{6,34} \mathrm{~A}$ two-temperature distribution in the electron energy spectrum was observed ${ }^{95}$ and attributed to a combination of two different acceleration mechanisms: (1) direct action of the laser field and (2) by the plasma wave. A similar two-temperature distribution was also observed ${ }^{113}$ to accompany a multicomponent spatial profile of the electron beam. In this case, electrons in the low energy range were observed to undergo an abrupt change in temperature, coinciding with the onset of extension of the laser channel due to selfguiding of the laser pulse, when the laser power or plasma density was varied. ${ }^{98}$ The fact that these same features were found in a simulation in which test electrons were injected into the self-consistent fields of a 3D plasma wave (without the presence of a laser pulse $)^{113}$ is inconsistent with interpretation that direct laser acceleration plays a significant role. Electrons have also been observed to be accelerated beyond the linear dephasing limit (the maximum energy electrons achieve before they outrun the wakefield and become decelerated), which was explained, using PIC simulations, to be the consequence of the electron-driven wakefields created by trapped electrons. ${ }^{114}$

Laser acceleration of electrons can be illustrated in Fig. $8 .^{4}$ Here, an intense laser interacts with a gas jet located inside a vacuum chamber. The laser crosses the picture from left to right and is focussed by a parabolic mirror (right side of the picture). The supersonic nozzle (shown in the middle 


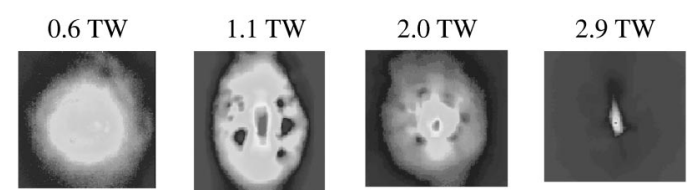

FIG. 9. Images of the spatial profiles of the electron beam measured by a ccd camera imaging a LANEX screen at a distance of $15 \mathrm{~cm}$ from the gas jet for various laser powers. The divergence angle of the beam decreases to a value of $\Delta \theta=1^{\circ}$ at a power of $2.9 \mathrm{TW}$, corresponding to a transverse geometrical emittance of just $\epsilon_{\perp} \leqslant 0.06 \pi \mathrm{mm}$ mrad.

of the picture) is positioned with micron accuracy with a 3 -axis micropositioner. The $e$-beam (up to $10^{10}$ electrons per shot) makes a small spot on a fluorescent (LANEX) screen (imaged with a CCD camera), shown in the upper left-hand corner of the picture. As shown in Fig. $9,{ }^{113}$ as the laser power increases, the divergence angle of the electron beam decreases. The lowest angle, $1^{\circ}$, obtained at the highest power, corresponds to a transverse geometrical emittance of $\epsilon_{\perp} \leqslant 0.06 \pi \mathrm{mm} \mathrm{mrad},{ }^{113}$ which is an order of magnitude lower than that from the best conventional electron gun. This may be because a large acceleration gradient decreases the time over which space charge can act to degrade the emittance.

Several groups ${ }^{115,116}$ have measured the plasma wave amplitude in the self-modulated regime as a function of time by means of collinear Thomson scattering and found that it decays in $\sim 50 \tau_{p}$. By direct measurement of ion waves, the modulational decay instability (in which electron plasma waves decay into ion waves) has been shown to play an important role in the damping of the plasma waves. ${ }^{117}$ The longitudinal spatial profile of the plasma wave has been measured by means of coherent Thomson sidescattering; it appears that - depending on the laser and plasma conditionsthe plasma wave can be localized to islands along the direction of laser propagation, due to multifocusing in the relativistic self-guiding process. ${ }^{118}$

The amplitude, wavelength, and resonant density of a wakefield in the resonant regime $\left(\tau \sim \tau_{p}\right)$ has been characterized using the technique of temporal interferometry. ${ }^{119,120}$ However this was done only for the tight-focusing case in which the laser spotsize is much smaller than the plasma wave wavelength $\left(r_{l} \ll \lambda_{p}\right)$ and thus the transverse wakefield was greater than the longitudinal wakefield.

The inverse free-electron laser mechanism has been invoked to explain the observation of accelerated electrons with 200 fs laser pulses. ${ }^{75}$ Electron acceleration without significant Raman scattering has also been observed ${ }^{102}$ with 30 fs laser pulses and acceleration near the resonant condition was found to be accompanied by multiple filamentation, the latter of which was also observed with longer duration pulses. ${ }^{121}$

Free electrons were reported to be accelerated in vacuum to $\mathrm{MeV}$ energies by a high-intensity subpicosecond laser pulse $\left(10^{19} \mathrm{~W} / \mathrm{cm}^{2}, 300 \mathrm{fs}\right){ }^{110}$ A subsequent discussion has helped to clarify the model used to explain the results. ${ }^{122-124}$

In numerical simulations ${ }^{125}$ as well as experiments, ${ }^{126}$ $\mathrm{MeV}$ energy electron beams have also been observed in the interactions of intense lasers with solid targets and to

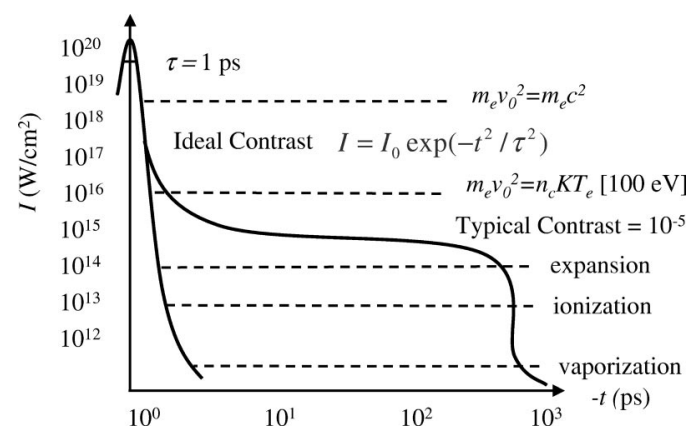

FIG. 10. Laser intensity versus time for two different laser-pulse contrasts, ideal Gaussian and typical. Also shown are the various mechanisms that occur in solid-target interactions; those that occur at low intensities are initiated significantly in advance of the peak of the pulse, which corresponds to time zero. This illustrates the need for high laser contrast.

propagate in both the forward and backward directions (with respect to the direction of laser propagation direction, which can also be in the specular direction).

\section{Proton acceleration and nuclear reactions}

Energetic ions from underdense plasmas were accelerated by an electrostatic sheath, which was created by charge displacement. Unlike earlier long-laser-pulse experiments, the displacement was due not to thermal expansion but to ponderomotive blow out. ${ }^{127,128}$ When a helium gas was used as the target, alpha particles were accelerated to several $\mathrm{MeV}$ in the direction orthogonal to the direction of laser propagation and along the direction of the maximum intensity gradient.

Several groups have reported the observation of ions originating from thin-film solid-density targets (or protons originating from monolayers of water on the target surface). Unlike previous long-pulse experiments, the protons were accelerated along the direction normal to the side of the target opposite to that upon which the laser was incident. For instance, Fig. 11 shows schematically the typical setup. Here, the laser (shown in the foreground) is focused with an off-axis parabola onto a thin-foil, held by a mesh that is positioned by a 3-axis micropositioner. A nuclear track detector, CR-39 (shown in white in the background), is used to detect the ions. An actual proton-produced pattern is shown. ${ }^{129}$ In another experiment, protons were observed to be emitted in ring patterns, the radii of which depend on the proton energy, which was explained by self-generated magnetic fields. ${ }^{130}$ Another recent result reported proton energies up to $60 \mathrm{MeV} .{ }^{131}$ The results of these experiments indicate that a large number of protons $\left(10^{13} p\right)$ can be accelerated, corresponding to current densities $\left(10^{8} \mathrm{~A} / \mathrm{cm}^{2}\right)$ at the source that are nine orders-of-magnitude higher than produced by cyclotrons, but with comparable transverse emittances $\left(\epsilon_{\perp}\right.$ $\leqslant 1.0 \pi \mathrm{mm} \mathrm{mrad}$ ). The high end of the energy spectrum typically has a sharp cutoff, but, like the electrons discussed in Sec. III B 3, is a continuum.

While the protons in several experiments originate from the front side of the target, ${ }^{129,130}$ in another, ${ }^{131}$ they originate from the back side. Evidence for a back-side origin comes from results obtained when wedge-shaped targets were used. 


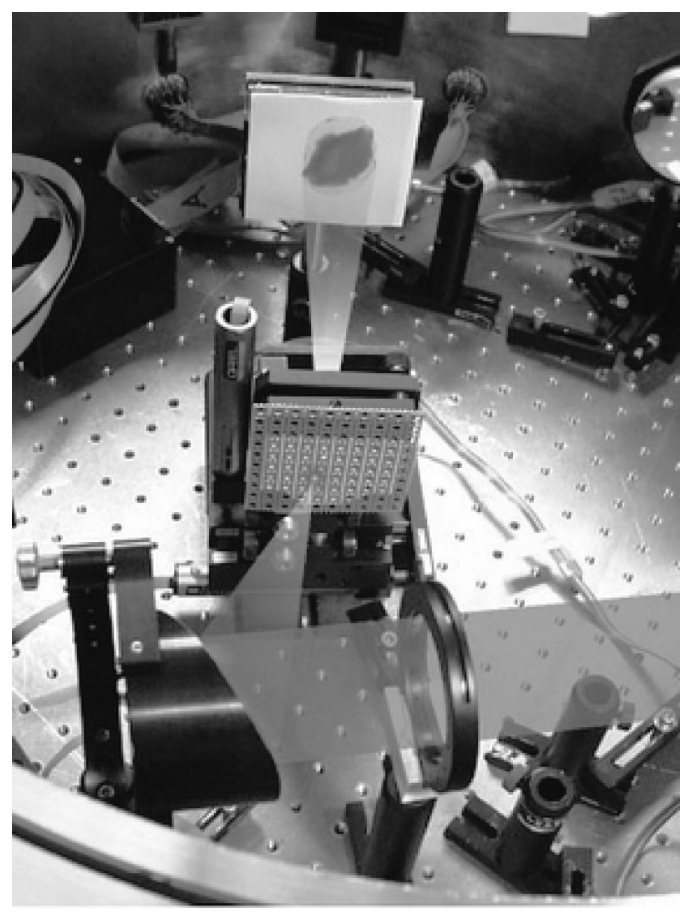

FIG. 11. Photograph of the typical setup used to observe the acceleration of ions. The laser (illustrated in the foreground) is focused with an off-axis parabola onto a thin foil, held by a mesh that is positioned by a 3-axis micropositioner. CR-39, a nuclear track detector (shown in white in the background) is used to detect the ions. An actual proton-produced pattern is shown.

The proton beam was observed to point in the direction normal to the back side of the target, which was not perpendicular to the front surface. On the other hand, evidence for a front-side origin comes from an experiment in which deuterium was coated on a thin film of mylar and a boron target was placed behind it. ${ }^{132}$ It was found that only when the deuterium was on the front side did the boron become activated by the reaction ${ }^{10} \mathrm{~B}(d, n){ }^{11} \mathrm{C}$. The production of radionuclides have also been used as an ion energy diagnostic. ${ }^{133}$

In another example of a nuclear reaction initiated by an intense laser, neutrons have been produced by the He fusion reaction $d(d, n)^{3}$ in the focus of $200 \mathrm{~mJ}, 160 \mathrm{fs}$ Ti:sapphire laser pulses on a deuterated polyethylene target. Optimizing the fast electron and ion generation by applying a welldefined prepulse led to an average rate of 140 neutrons per shot. ${ }^{134}$ Also, bright $x$-rays from solid-target interactions have created isotopes of high- $Z$ metals by means of photofission. ${ }^{135,136}$ Laser-accelerated electron energies and angular distributions have been inferred from analyzing $(\gamma$, $n)$ and $(\gamma, 2 n)$ reactions in composite $\mathrm{Pb} / \mathrm{Cu}$ targets ${ }^{112}$ and in $\mathrm{Ta} / \mathrm{Cu}$ targets. ${ }^{137}$ Positrons were created by colliding laseraccelerated electrons with a tungsten target. ${ }^{138}$

\section{Summary}

Presented here is partial list of some of the recent experimental advances in high-field science discussed in greater detail above: (1) Thomson scattering from the figure-eight motion of relativistic electrons; (2) self-guiding of intense laser light $\left(a_{0} \sim 1\right)$ over distances extending up to 5 times the

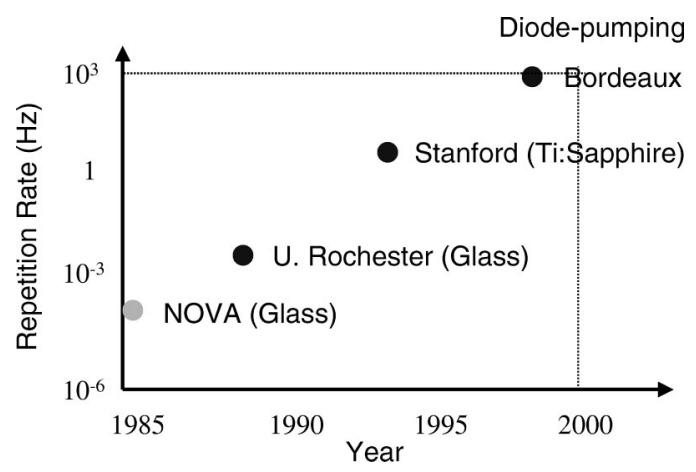

FIG. 12. The repetition rate of table-top terawatt lasers has increased exponentially in the last decade, reaching $1 \mathrm{kHz}$, where the use signal averaging to significantly reduce noise is now possible. Note that the NOVA laser system, which had TW level power but much greater size than table-top, is shown only for comparison.

Rayleigh range $\left(L_{R}\right)$; (3) guiding in preformed channels over distances up to $20 L_{R}$, but for $a_{0} \ll 1$; (4) well-characterized wakefield amplitudes; (5) guided acceleration of electrons to $\mathrm{MeV}$ energies with extremely low transverse emittance, but large energy spreads; (6) absorption by vacuum heating; (7) $\mathrm{MeV}$ energy ions; (8) production of hard $\mathrm{x}$-rays, isotopes, and positrons.

\section{PROSPECTS AND APPLICATIONS}

Some of the advances on the horizon in the area of theory and simulation include (1) improved 3D codes; (2) massively parallel processing; (3) 3D visualization; and (4) fluid/particle hybrid models.

Advances in laser technology are making new physical phenomena accessible and improving the accuracy of high field measurements. For instance, as shown in Fig. 12, the repetition rate of terawatt-class lasers has increased exponentially in the last decade, making it easier to use signal averaging to increase signal-to-noise levels. Taking full advantage of the intrinsic bandwidth of Ti:sapphire, the pulse duration of terawatt lasers is also decreasing, approaching the single-cycle limit. With adaptive optics such as deformable mirrors (a technology borrowed from astronomy), these lasers can also now be focused almost to the diffraction limit (a single wavelength). ${ }^{139}$ The intensity of table-top lasers will increase significantly with the anticipated use of dielectric gratings, which have higher damage thresholds than the gold-coated gratings that are in current use. ${ }^{140}$

Because of either imperfect compression or amplified spontaneous emission, background light accompanies the short intense pulse at the focus. Thus, as laser intensities increase, so does the need for higher laser contrast (the ratio of the peak intensity to the background light intensity). Plotted in Fig. 10 is the laser intensity versus time for two different laser-pulse contrasts, an ideal Gaussian shape and a typical pulse (contrast of $10^{5}$ ). Also shown are the various mechanisms that occur in solid-target interactions at various intensities. The peak of a high intensity laser pulse can be orders of magnitude above the thresholds of, and arrive significantly after, the onset of plasma creation and expansion. Under such conditions, the high intensity portion of the laser 
pulse will deposit its energy at the critical density of a longdensity-scalelength plasma rather than directly at solid density. In order to mitigate this problem for experiments where a short scalelength is required, the laser contrast is being improved by the use of frequency doubling, saturable absorbers $^{141}$ or frequency modulators to correct for highorder phase aberrations. ${ }^{142}$ The latter technology is also permitting the generation of arbitrarily shaped pulses.

Compression and amplification of laser pulses with plasma gratings ${ }^{143}$ might also someday increase the maximum power density of intense lasers, which in chirped pulse amplification systems is limited by the material damage of the final metal or dielectric grating. As the sophistication of laser technology increases due to these improvements, some of the following exciting experimental possibilities can be imagined.

At the higher intensities that should be achievable in the near future $\left(I \simeq 10^{24} \mathrm{~W} / \mathrm{cm}^{2}, a_{0} \sim 1000\right)$, we can look forward to the regime in which even protons begin to quiver relativistically. It is predicted that protons can be accelerated to relativistic velocities in plasma wakefields at much lower intensities $\left(I \simeq 10^{21} \mathrm{~W} / \mathrm{cm}^{2}, a_{0} \sim 30\right) .{ }^{69}$ At intensities exceeding $I \simeq 10^{20} \mathrm{~W} / \mathrm{cm}^{2}, a_{0} \sim 10$, positrons will be produced more rapidly than they annihilate, making it possible to create a dense electron-positron plasma. ${ }^{86}$ Such an exotic plasma exists at the horizons of black holes and is thus relevant to astrophysics.

Proof-of-principle experiments are underway at various laboratories to use multiple synchronized laser pulses ${ }^{142}$ to simultaneously channel-guide intense laser pulses ${ }^{103-106}$ and coherently control wakefields ${ }^{29,60,61}$ and electron injection. ${ }^{38,69-73}$ The near-term milestone goal is to accelerate electrons monoenergetically up to an energy of $1 \mathrm{GeV}$ in a single $1 \mathrm{~cm}$ long plasma channel. This could be the first stage of a multiply staged accelerator that might someday achieve energies relevant to high-energy physics. It could also be important for applications requiring low longitudinal emittance, such as free-electron lasers.

A promising avenue for the production of $\mathrm{x}$ rays is based on the use of Compton scattering. ${ }^{144,145}$ Upon scattering with an electron beam, laser light can be upshifted due to a relativistic boost by a factor of $4 \gamma^{2}$, which for electrons accelerated to $30 \mathrm{MeV}$ corresponds to a factor of 10000 . Thus a 1 $\mathrm{eV}$ photon can be upshifted to $10 \mathrm{keV}$. Such coherent, ultrashort duration and energetic sources could enable ultrafast imaging on the atomic scale. If the source of the electron beam were a laser accelerator, the footprint of this synchrotron-like device would be small enough for it to fit in a university laboratory. Several labs have already used shortpulse incoherent $x$ rays generated from solid-target interactions to study time-resolved ultrafast phenomena, such as melting. ${ }^{146-148}$

It was demonstrated that there are a sufficient number of electrons accelerated by laser-plasma accelerators to conduct pulsed radiolysis. ${ }^{149}$ Time-resolved radio-biological studies with laser accelerated protons are also feasible.

It has been shown theoretically that a laser-induced burst of hot electrons or ions could be used as a spark to ignite a thermonuclear reaction with inertial confinement fusion in the so-called fast-ignitor laser fusion concept. In its original conception, a short but energetic laser pulse would drill through the under-dense plasma that surrounds the fusion core and a second shorter pulse would deposit energy in the core in the form of $\mathrm{MeV}$ electrons. In so doing, it would relax the otherwise stringent requirements on energy and symmetry of the long-pulse-duration heating and compression pulses. ${ }^{150,151}$ More recently, the use of a short pulse of ions for the ignitor has also been discussed. ${ }^{152,153}$

If protons could be laser accelerated to $70-160 \mathrm{MeV}$ energies, they could be useful for proton therapy, which is now limited by the extraordinary expense of cyclotrons or synchrotrons and the large magnets required to transport the proton beams to the patient. Protons are superior to other forms of ionizing radiation for cancer treatment because of less straggling and their ability to deposit their energy over a narrower depth range.

\section{ACKNOWLEDGMENTS}

The author acknowledges the support of the Department of Energy and the National Science Foundation and thanks all of the many scientists who contributed data and ideas for this paper, in particular E. Esarey, Y. Y. Lau, and A. Maksimchuk for their helpful comments.

${ }^{1}$ P. Maine, D. Strickland, P. Bado, M. Pessot, and G. Mourou, IEEE J. Quantum Electron. 24, 398 (1988).

${ }^{2}$ M. D. Perry and G. Mourou, Science 264, 917 (1994), and references therein.

${ }^{3}$ G. A. Mourou, C. P. J. Barty, and M. D. Perry, Phys. Today 51, 22 (1998), and references therein.

${ }^{4}$ D. Umstadter, C. Barty, M. Perry, and G. Mourou, Opt. Photonics News 9, 40 (1998), and references therein.

${ }^{5}$ D. Umstadter and T. Norris, Eds., IEEE J. Quantum Electron. 33, 1878 (1997), and references therein.

${ }^{6}$ E. Esarey, P. Sprangle, J. Krall, and A. Ting, IEEE Trans. Plasma Sci. PS-24, 252 (1996), and references therein.

${ }^{7}$ P. Gibbon and E. Forster, Plasma Phys. Controlled Fusion 38, 769 (1996), and references therein.

${ }^{8}$ C. J. Joshi and P. B. Corkum, Phys. Today 48, 36 (1995), and references therein.

${ }^{9}$ E. G. Gamaly, Laser Part. Beams 12, 185 (1994), and references therein.

${ }^{10}$ G. Mourou and D. Umstadter, Phys. Fluids B 4, 2315 (1992), and references therein.

${ }^{11}$ B., Luther-Davies, E. G. Gamaly, Y. Wang, A. V. Rode, and V. T. Tikhonchuk, Sov. J. Quantum Electron. 22, 289 (1992), and references therein.

${ }^{12}$ E. M. Campbell, Phys. Fluids B 4, 3781 (1992), and referernces therein.

${ }^{13}$ N. H. Burnett, H. A. Baldis, M. C. Richardson, and G. D. Enright, Appl. Phys. Lett. 31, 172 (1977).

${ }^{14}$ I. V. Pogorelsky, AIP Conf. Proc. 426, 415 (1998).

${ }^{15}$ S. Ya. Tochitsky, R. Narang, C. Filip, C. E. Clayton, K. A. Marsh, and C. Joshi, Opt. Lett. 24, 1717 (1999).

${ }^{16}$ L. D. Landau and E. M. Lifshits, Teoriia Polia (Nauka, Moskva, 1948), translated The Classical Theory of Fields (Pergamon, Oxford, 1975).

${ }^{17}$ V. L. Ginzburg, Key Problems of Physics and Astrophysics (MIR, Moscow, 1978).

${ }^{18}$ A. G. Litvak, Zh. Eksp. Teor. Fiz. 57, 629 (1969); Sov. Phys. JETP 30, 344 (1969).

${ }^{19}$ C. Max, J. Arons, and A. B. Langdon, Phys. Rev. Lett. 33, 209 (1974).

${ }^{20}$ G. Z. Sun, E. Ott, Y. C. Lee, and P. Guzdar, Phys. Fluids 30, 526 (1987).

${ }^{21}$ D. C. Barnes, T. Kurki-Suonio, and T. Tajima, IEEE Trans. Plasma Sci. PS-15, 154 (1987).

${ }^{22}$ A. B. Borisov, A. V. Borovskiy, O. B. Shiryaev, V. V. Korobkin, A. M. Porkhorov, J. C. Solem, T. S. Luk, K. Boyer, and C. K. Rhodes, Phys. Rev. A 45, 5830 (1992). 
${ }^{23}$ P. Sprangle, E. Esarey, J. Krall, and G. Joyce, Phys. Rev. Lett. 69, 2200 (1992).

${ }^{24}$ T. M. Antonsen, Jr. and P. Mora, Phys. Rev. Lett. 69, 2204 (1992).

${ }^{25}$ N. E. Andreev, L. M. Gorbunov, V. I. Kirsanov, A. Pogosova, and R. R. Ramazashvili, Pis'ma Zh. Eksp. Teor. Fiz. 55, 551 (1992) [JETP Lett. 55, 571 (1992)].

${ }^{26}$ T. Tajima and J. M. Dawson, Phys. Rev. Lett. 43, 267 (1979).

${ }^{27}$ P. Chen, Part. Accel. 20, 171 (1985).

${ }^{28}$ L. M. Gorbunov and V. I. Kirsanov, Zh. Eksp. Teor. Fiz. 93, 509 (1987); Sov. Phys. JETP 66, 290 (1987).

${ }^{29}$ D. Umstadter, J. K. Kim, and E. Dodd, Phys. Rev. Lett. 72, 1224 (1994).

${ }^{30}$ J. B. Rosenzweig, D. B. Cline, B. Cole, H. Figueroa, W. Gai, R. Konecny, J. Norem, P. Schoessow, and J. Simpson, Phys. Rev. Lett. 61, 98 (1988).

${ }^{31}$ C. E. Clayton, C. Joshi, C. Darrow, and D. Umstadter, Phys. Rev. Lett. 54, 2343 (1985)

${ }^{32}$ Y. Kitagawa, T. Matsumoto, T. Minamihata et al., Phys. Rev. Lett. 68, 48 (1992).

${ }^{33}$ C. E. Clayton, K. A. Marsh, A. Dyson, M. Everett, A. Lai, W. P. Leemans, R. Williams, and C. Joshi, Phys. Rev. Lett. 70, 37 (1993).

${ }^{34}$ P. Bertrand, A. Ghizzo, S. J. Karttunen, T. J. H. Pattikangas, R. R. E. Salomaa, and M. Shoucri, Phys. Rev. E 49, 5656 (1994).

${ }^{35}$ A. Modena, Z. Najmudin, A. E. Dangor, C. E. Clayton, K. A. Marsh, C. Joshi, V. Malka, C. B. Carrow, C. Danson, D. Neely, and F. N. Walsh, Nature (London) 377, 606 (1995).

${ }^{36}$ C. L. Moore, A. Ting, K. Krushelnick, E. Esarey, R. F. Hubbard, B. Hafizi, H. R. Burris, C. Manka, and P. Sprangle, Phys. Rev. Lett. 79, 3909 (1997).

${ }^{37}$ S. V. Bulanov, F. Pegoraro, A. M. Pukhov, and A. S. Sakharov, Phys. Rev. Lett. 78, 4205 (1997).

${ }^{38}$ D. Umstadter, J. K. Kim, and E. Dodd, Phys. Rev. Lett. 76, 2073 (1996).

${ }^{39}$ M. Borghesi, A. J. Mackinnon, R. Gaillard, O. Willi, A. Pukhov, and J. Meyer-ter-Vehn, Phys. Rev. Lett. 80, 5137 (1998).

${ }^{40}$ B. Bhattacharyya, P. Mulser, and U. Sanyal, Phys. Lett. A 249, 4 (1999); 249, 324 (1999)

${ }^{41}$ F. Brunel, Phys. Rev. Lett. 59, 52 (1987).

${ }^{42}$ N. H. Burnett and G. D. Enright, IEEE J. Quantum Electron. 26, 1797 (1990).

${ }^{43}$ B. Bezzerides, R. D. Jones, and D. W. Forslund, Phys. Rev. Lett. 49, 202 (1982).

${ }^{44}$ X. Liu and D. Umstadter, Phys. Rev. Lett. 69, 1935 (1992).

${ }^{45}$ M. P. Kalashnikov, P. V. Nickles, Th. Schlegel, M. Schnuerer, F. Billhardt, I. Will, and W. Sandner, and N. N. Demchenko, Phys. Rev. Lett. 73, 260 (1994)

${ }^{46}$ S. C. Wilks, W. L. Kruer, M. Tabak, and A. B. Langdon, Phys. Rev. Lett. 69, 1383 (1992)

${ }^{47}$ A. S. Sakharov and V. I. Kirsanov, Phys. Plasmas 4, 3382 (1997).

${ }^{48}$ H. C. Barr, P. Mason, and D. M. Parr, Phys. Plasmas 7, 2604 (2000).

${ }^{49}$ E. Esarey, C. B. Schroeder, B. A. Shadwick, J. S. Wurtele, and W. P. Leemans, Phys. Rev. Lett. 84, 3081 (2000).

${ }^{50}$ B. J. Duda and W. B. Mori, Phys. Rev. E 61, 1925 (2000).

${ }^{51}$ A. Pukhov and J. Meyer-ter-Vehn, Phys. Rev. Lett. 76, 3975 (1996).

${ }^{52}$ A. Pukhov and J. Meyer-ter-Vehn, Phys. Rev. Lett. 79, 2686 (1997).

${ }^{53}$ C. Ren, R. G. Hemker, R. Fonseca, B. J. Duda, and W. B. Mori, Phys. Rev. Lett. 85, 2124 (2000).

${ }^{54}$ P. Sprangle, B. Hafizi, J. R. Renano, R. F. Hubbard, A. Ting, A. Zigler, and T. M. Antonsen, Jr., Phys. Rev. Lett. 85, 5110 (2000).

${ }^{55}$ B. Ritchie and C. D. Decker, Phys. Rev. E 57, 4645 (1998).

${ }^{56}$ E. A. Startsev and C. J. McKinstrie, Phys. Rev. E 55, 7527 (1997).

${ }^{57}$ G. Shvets, N. J. Fisch, A. Pukhov, and J. Meyer-ter-Vehn, Phys. Rev. E 60, 2218 (1999).

${ }^{58}$ G. Shvets, N. J. Fisch, and J.-M. Rax, Phys. Plasmas 3, 1109 (1996).

${ }^{59}$ K.-C. Tzeng and W. B. Mori, Phys. Rev. Lett. 81, 104 (1998).

${ }^{60}$ A. Spitkovsky and P. Chen (unpublished).

${ }^{61}$ Rundquist, A. R., S. P. LeBlanc, E. W. Gaul, S. Cheshkov, F. B. Grigsby, T. T. Tajima, and M. C. Downer, "Optimization of the laser wakefield," Proceedings of Advanced Accelerator Conference IX (in press).

${ }^{62}$ C. D. Decker, W. B. Mori, and T. Katsouleas, Phys. Rev. E 50, R3338 (1994).

${ }^{63}$ N. E. Andreev, L. M. Gorbunov, and S. V. Kuznetsov, IEEE Trans. Plasma Sci. PS-24, 448 (1996).

${ }^{64}$ K.-C. Tzeng, W. B. Mori, and T. Katsouleas, Phys. Rev. Lett. 79, 5258 (1997).

${ }^{65}$ E. Esarey, B. Hafizi, R. F. Hubbard, and A. Ting, Phys. Rev. Lett. 80, 5552 (1998)
${ }^{66}$ S. Wilks, T. Katsouleas, J. M. Dawson, P. Chen, and J. J. Su, IEEE Trans. Plasma Sci. PS-15, 210 (1987).

${ }^{67}$ P. Mora, J. Appl. Phys. 71, 2087 (1992).

${ }^{68}$ L. Gorbunov, P. Mora, and T. M. Antonsen, Jr., Phys. Rev. Lett. 76, 2495 (1996).

${ }^{69}$ S. V. Bulanov, V. A. Vshivkov, G. I. Dudnikova, T. Z. Esirkepov, F. Califano, F. F. Kamenets, T. V. Liseikina, N. M. Naumova, and F. Pegoraro, Plasma Phys. Rep. 25, 701 (1999).

${ }^{70}$ E. Esarey, R. F. Hubbard, W. P. Leemans, A. Ting, and P. Sprangle, Phys. Rev. Lett. 79, 2682 (1997).

${ }^{71}$ B. Rau, T. Tajima, and H. Hojo, Phys. Rev. Lett. 78, 3310 (1997).

${ }^{72}$ R. G. Hemker, K-C. Tzeng, W. B. Mori, C. E. Clayton, and T. Katsouleas, Phys. Rev. E 57, 5920 (1998).

${ }^{73}$ C. I. Moore, A. Ting, S. J. McNaught, J. Qiu, H. R. Burris, and P. Sprangle, Phys. Rev. Lett. 82, 1688 (1999).

${ }^{74}$ A. Pukhov, Z. M. Seng, and J. Meyer-ter-Vehn, Phys. Plasmas 6, 2847 (1999).

${ }^{75}$ C. Gahn, G. D. Tsakiris, A. Pukhov, J. Meyer-ter-Vehn, G. Pretzler, P. Thirolf, and K. J. Witte, Phys. Rev. Lett. 83, 4772 (1999).

${ }^{76}$ B. Quesnel and P. Mora, Phys. Rev. E 58, 3719 (1998).

${ }^{77}$ A. L. Troha, J. R. Van Meter, E. C. Landahl, R. M. Alvis, Z. A. Unterberg, K. Li, N. C. Luhmann, Jr., A. K. Kerman, and F. V. Hartemann, Phys. Rev. E 60, 926 (1999).

${ }^{78}$ H. Ruhl, "3D PIC simulations of proton acceleration from thin spherical targets," Plasma Phys. Rep. (in press).

${ }^{79}$ S. C. Wilks, A. B. Langdon, T. E. Cowan, M. Roth, M. Singh, S. Hatchett, M. H. Key, D. Pennington, A. MacKinnon, and R. A. Snavely, Phys. Plasmas 8, 542 (2001).

${ }^{80}$ S. V. Bulanov, T. Zh. Esirkepov, F. Califano et al., JETP Lett. 71, 407 (2000).

${ }^{81}$ Z.-M. Sheng, Y. Sentoku, K. Kima, J. Zhang, W. Yu, and J. Meyer-terVehn, Phys. Rev. Lett. 85, 5340 (2000).

${ }^{82}$ P. Gibson, Phys. Rev. Lett. 76, 50 (1996).

${ }^{83}$ W. Yu, M. Y. Yu, J. Zhang, and Z. Xu, Phys. Rev. E 57, R2531 (1998).

${ }^{84}$ R. Lichters, J. Meyer-ter-Vehn, and A. Pukhov, Phys. Plasmas 3, 3425 (1996).

${ }^{85}$ I. Yu. Kostyukov and J. M. Rax, Phys. Rev. Lett. 83, 2206 (1999).

${ }^{86}$ E. P. Liang, S. C. Wilks, and M. Tabak, Phys. Rev. Lett. 81, 4887 (1999).

${ }^{87}$ S. Y. Chen, A. Maksimchuk, and D. Umstadter, Nature (London) 396, 653 (1998).

${ }^{88}$ S.-Y. Chen, A. Maksimchuk, E. Esarey, and D. Umstadter, Phys. Rev. Lett. 84, 5528 (2000)

${ }^{89}$ C. J. Moore, J. P. Knauer, and D. D. Meyerhofer, Phys. Rev. Lett. 74, 2439 (1995).

${ }^{90}$ P. Norreys, M. Zepf, S. Moustaizis et al., Phys. Rev. Lett. 76, 1832 (1996).

${ }^{91}$ M. K. Grimes, A. R. Rundquist, Y.-S. Lee, and M. C. Downer, Phys. Rev. Lett. 82, 4010 (1999).

${ }^{92}$ D. L. Burke, R. C. Field, G. Horton-Smith et al., Phys. Rev. Lett. 79, 1626 (1997).

${ }^{93}$ A. B. Borisov, A. V. Borovskii, V. V. Korobkin, A. M. Prokhorov, J. C. Solem, T. S. Luk, K. Boyer, and C. K. Rhodes, Phys. Rev. Lett. 68, 2309 (1992).

${ }^{94}$ P. Monot, T. Auguste, P. Gibbon, F. Jakober, G. Mainfray, A. Dulieu, M. Louis-Jacquet, G. Malka, and J. L. Miquel, Phys. Rev. Lett. 74, 2953 (1995).

${ }^{95}$ G. Malka, J. Fuchs, F. Amiranoff et al., Phys. Rev. Lett. 79, 2053 (1997).

${ }^{96}$ M. Borghesi, A. J. MacKinnon, L. Barringer, R. Gaillard, L. A. Gizzi, C. Meyer, O. Willi, A. Pukhov, and J. Meyer-ter-Vehn, Phys. Rev. Lett. 78, 879 (1997)

${ }^{97}$ J. Fuchs, G. Malka, J. C. Adam et al., Phys. Rev. Lett. 80, 1658 (1998).

${ }^{98}$ R. Wagner, S.-Y. Chen, A. Maksimchuk, and D. Umstadter, Phys. Rev. Lett. 78, 3125 (1997).

${ }^{99}$ K. Krushelnick, A. Ting, C. I. Moore, H. R. Burris, E. Esarey, P. Sprangle, and M. Baine, Phys. Rev. Lett. 78, 4047 (1997).

${ }^{100}$ S.-Y. Chen, G. S. Sarkisov, A. Maksimchuk, R. Wagner, and D. Umstadter, Phys. Rev. Lett. 80, 2610 (1998).

${ }^{101}$ P. E. Young and P. R. Bolton, Phys. Rev. Lett. 75, 4173 (1995).

${ }^{102}$ X. Wang, M. Krishnan, N. Saleh, H. Wang, and D. Umstadter, Phys. Rev. Lett. 84, 5324 (2000).

${ }^{103}$ S. P. Nikitin, I. Alexeev, J. Fan, and H. M. Milchberg, Phys. Rev. E 59, R3839 (1999).

${ }^{104}$ E. W. Gaul, S. P. Le Blanc, A. R. Rundquist, R. Zgadzaj, H. Langhoff, and M. C. Downer, Appl. Phys. Lett. 77, 4112 (2000). 
${ }^{105}$ P. Volfbeyn, E. Esarey, and W. P. Leemans, Phys. Plasmas 6, 2269 (1999).

${ }^{106}$ R. F. Hubbard, Y. Ehrlich, D. Kaganovich, C. Cohen, C. I. Moore, P. Sprangle, A. Ting, and A. Zigler, Advanced Accelerator Concepts, Eighth Workshop, 6-11 July 1998, Baltimore, MD, AIP Conf. Proc. No. 472 (American Institute of Physics, Melville, NY, 1999), pp. 394-403.

${ }^{107}$ K. Takahashi, R. Kodama, K. A. Tanaka, H. Hashimoto, Y. Kato, K. Mima, F. A. Weber, T. W. Barbee, Jr., and L. B. Da Silva, Phys. Rev. Lett. 84, 2405 (2000)

${ }^{108}$ J. Fuchs, J. C. Adam, F. Amiranoff et al., Phys. Rev. Lett. 80, 2326 (1998).

${ }^{109}$ D. Umstadter, S.-Y. Chen, A. Maksimchuk, G. Mourou, and R. Wagner, Science 273, 472 (1996).

${ }^{110}$ G. Malka, E. Lefebvre, and J. L. Miquel, Phys. Rev. Lett. 78, 3314 (1997).

${ }^{111}$ A. Ting, C. I. Moore, K. Krushelnick, C. Manka, E. Esarey, P. Sprangle, R. Hubbard, H. R. Burris, R. Fischer, and M. Baine, Phys. Plasmas 4, 1889 (1997).

${ }^{112}$ W. P. Leemans, D. Rodgers, P. E. Catravas, C. G. R. Geddes, G. Fubiani, E. Esarey, B. A. Shadwick, R. Donahue, and A. Smith, Phys. Plasmas 8, 2510 (2001).

${ }^{113}$ S.-Y. Chen, M. Krishnan, A. Maksimchuk, R. Wagner, and D. Umstadter, Phys. Plasmas 6, 4739 (1999).

${ }^{114}$ D. Gordon, K. C. Tzeng, C. E. Clayton, A. E. Dangor, V. Malka, K. A. Marsh, A. Modena, and W. B. Mora, Phys. Rev. Lett. 80, 2133 (1998).

${ }^{115}$ A. Ting, K. Krushelnick, C. I. Moore, H. R. Burris, E. Esarey, J. Krall, and P. Sprangle, Phys. Rev. Lett. 77, 5377 (1996).

${ }^{116}$ S. P. Le Blanc, M. C. Downer, R. Wagner, S.-Y. Chen, A. Maksimchuk, G. Mourou, and D. Umstadter, Phys. Rev. Lett. 77, 5381 (1996).

${ }^{117}$ S.-Y. Chen, M. Krishnan, A. Maksimchuk, and D. Umstadter, Phys. Plasmas 7, 403 (2000).

${ }^{118}$ C. E. Clayton, K. C. Tzeng, D. Gordon et al., Phys. Rev. Lett. 81, 100 (1998).

${ }^{119}$ J. R. Marques, J. P. Geindre, F. Amiranoff, P. Audebert, J. C. Gauthier, A. Antonetti, and G. Grillon, Phys. Rev. Lett. 76, 3566 (1996).

${ }^{120}$ C. W. Siders, S. P. Le Blanc, D. Fisher, T. Tajima, M. C. Downer, A. Babine, A. Stepanov, and A. Sergeev, Phys. Rev. Lett. 76, 3570 (1996).

${ }^{121}$ P. E. Young and P. R. Bolton, Phys. Rev. Lett. 77, 4556 (1996).

${ }^{122}$ K. T. McDonald, Phys. Rev. Lett. 80, 1350 (1998).

${ }^{123}$ P. Mora and B. Quesnel, Phys. Rev. Lett. 80, 1351 (1998).

${ }^{124}$ E. Lefebvre, G. Malka, and J.-L. Miquel, Phys. Rev. Lett. 80, 1352 (1998).

${ }^{125}$ Wei Yu, V. Bychenkov, Y. Sentoku, M. Y. Yu, Z. M. Sheng, and K. Mima, Phys. Rev. Lett. 85, 570 (2000).

${ }^{126}$ R. Kodama, K. A. Tanaka, Y. Sentoku, T. Matsushita, K. Takahashi, H. Fujita, Y. Kitagawa, Y. Kato, T. Yamanaka, and K. Mima, Phys. Rev. Lett. 84, 674 (2000).

${ }^{127}$ G. S. Sarkisov, V. Yu. Bychenkov, V. N. Novikov, V. T. Tikhonchuk, A. Maksimchuk, S. Y. Chen, R. Wagner, G. Mourou, and D. Umstadter, Phys. Rev. E 59, 7042 (1999).

${ }^{128}$ K. Krushelnick, E. L. Clark, Z. Najmudin et al., Phys. Rev. Lett. 83, 737 (1999).
${ }^{129}$ A. Maksimchuk, S. Gu, K. Flippo, D. Umstadter, and V. Y. Bychenkov, Phys. Rev. Lett. 84, 4108 (2000).

${ }^{130}$ E. L. Clark, K. Krushelnick, J. R. Davies et al., Phys. Rev. Lett. 84, 670 (2000).

${ }^{131}$ R. A. Snavely, M. H. Key, S. P. Hatchett et al., Phys. Rev. Lett. 85, 2945 (2000).

${ }^{132}$ K. Nemoto, A. Maksimchuk, S. Banerjee, K. Flippo, G. Mourou, D. Umstadter, and V. Yu. Bychenkov, Appl. Phys. Lett. 78, 595 (2001).

${ }^{133}$ M. L. K. Santala, M. Zepf, F. N. Berg et al., Appl. Phys. Lett. 78, 19 (2001).

${ }^{134}$ G. Pretzler, A. Saemann, A. Pukhov et al., Phys. Rev. E 58, 1165 (1998).

${ }^{135}$ T. E. Cowan, A. W. Hunt, T. W. Phillips et al., Phys. Rev. Lett. 84, 903 (2000).

${ }^{136}$ K. W. D. Ledingham, I. Spencer, T. McCanny et al., Phys. Rev. Lett. 84, 899 (2000).

${ }^{137}$ M. I. K. Santala, Z. Najmudin, E. L. Clark, M. Tatarakis, K. Krushelnick, A. E. Dangor, V. Malka, J. Faure, R. Allott, and R. J. Clarke, Phys. Rev. Lett. 86, 1227 (2001).

${ }^{138}$ C. Gahn, G. D. Tsakiris, G. Pretzler et al., Phys. Lett., C 77, 2662 (2000).

${ }^{139}$ O. Albert, H. Wang, D. Liu, Z. Chang, and G. Mourou, Opt. Lett. 25, 1125 (2000).

${ }^{140}$ B. W. Shore, M. D. Perry, J. A. Britten, R. D. Boyd, M. D. Feit, H. T. Nguyen, R. Chow, and G. E. Loomis, J. Opt. Soc. Am. A 14, 1124 (1997).

${ }^{141}$ J. Italani, J. Faure, M. Nantel, G. Mourou, and S. Watanabe, Opt. Commun. 148, 70 (1998)

${ }^{142}$ M. M. Wefers and K. A. Nelson, J. Opt. Soc. Am. B 12, 1343 (1995).

${ }^{143}$ V. M. Malkin, G. Shvets, and N. J. Fisch, Phys. Plasmas 7, 2232 (2000).

${ }^{144}$ W. P. Leemans, R. W. Schoenlein, P. Volfbeyn, A. H. Chin, T. E. Glover, P. Balling, M. Zolotorev, K. J. Kim, S. Chattopadhyay, and C. V. Shank, Phys. Rev. Lett. 77, 4182 (1996).

${ }^{145}$ I. V. Pogorelsky, T. Ben-Zvi, T. Hirose et al., Phys. Rev. ST Accel. Beams 3, 090702 (2000).

${ }^{146}$ J. Workman, M. Nantel, A. Maksimchuk, and D. Umstadter, Appl. Phys. Lett. 70, 312 (1997).

${ }^{147}$ C. Rischel, A. Rousse, I. Uschmann, P. A. Albouy, J. P. Geindre, P. Audebert, J. C. Gauthier, E. Forster, J. L. Martin, and A. Antonetti, Nature (London) 390, 490 (1997).

${ }^{148}$ C. W. Siders, A. Cavalleri, K. Sokolowski-Tinten, C. Toth, T. Guo, M. Kammler, M. H. van-Hoegen, K. R. Wilson, D. van-der-Linde, and C. P. J. Barty, Science 286, 1340 (1999).

${ }^{149}$ N. Saleh, K. Flippo, K. Nemoto, D. Umstadter, R. A. Crowell, C. D. Jonah, and A. D. Trifunac, Rev. Sci. Instrum. 71, 2305 (2000).

${ }^{150}$ M. Tabak, J. Hammer, M. E. Glinsky, W. L. Kruer, S. C. Wilks, J. Woodworth, E. M. Campbell, M. D. Perry, and R. J. Mason, Phys. Plasmas 1, 1626 (1994)

${ }^{151}$ C. Deutsch, H. Furukawa, K. Mima, M. Murakami, and K. Nishihara, Phys. Rev. Lett. 77, 2483 (1996).

${ }^{152}$ M. Roth, T. E. Cowan, M. H. Key et al., Phys. Rev. Lett. 86, 436 (2001).

${ }^{153}$ V.Yu. Bychenkov, W. Rozmus, A. Maksimchuk, D. Umstadter, and C. E. Capjack, "Fast ignitor concept with light ions," Commun. Plasma Phys. Control Fusion (in press). 